\title{
Publications pattern of dermatology research in Saudi Arabia
}

\author{
Fahad M. Alsaif, MD, Sarah F. Alsukait, MD, Alaa B. Alsaad, MD, Nuha A. Alfurayh, MD, Rama A. Alhallaf, MD, \\ Sara A. Alhaddab, MD, Hend M. Alotaibi, MD.
}

\begin{abstract}
الأهداف : تحليل كمية وخصائص الأبحاث المنشوره للأمراض الجلدية في المملكة العربية السعودية على مر السمدية وخصائل الأبحاث.

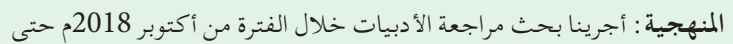

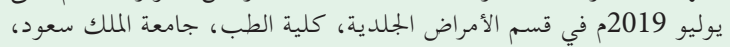

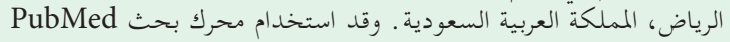

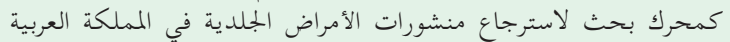

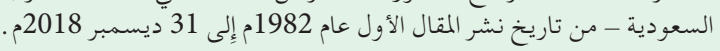

النتائج : اشتملت الدراسة على 500 بحث من منشور . تمت كتابة ثلثيها بين

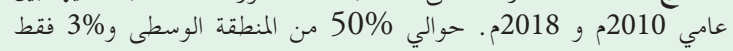

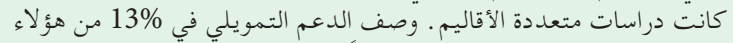

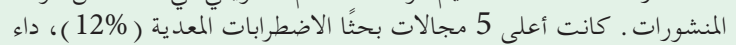

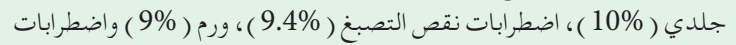

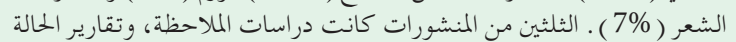

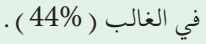

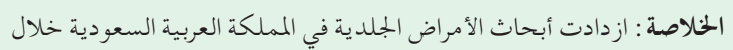

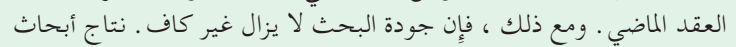

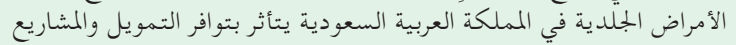

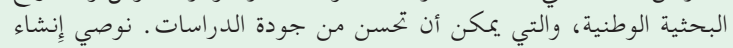

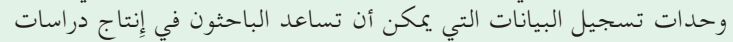

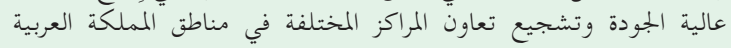

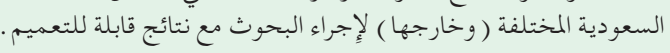

Objectives: To analyze the quantity and characteristics of Saudi Arabia's (SA's) dermatology research publications throughout the years.

Methods: A literature search was conducted between October 2018 and July 2019 in the Dermatology Department, College of Medicine, King Saud University, Riyadh, Saudi Arabia. PubMed was used as a search engine, to retrieve dermatology-related publications in SA - from the date of the first article publication in 1982 to December 31, 2018.

Results: Five hundred publications were included. Two-thirds of them were written between 2010 and 2018. Approximately 50\% were from the central region and only $3 \%$ were multiregional studies.
Funding support was described in 13\% of these publications. The top 5 most-researched fields were infectious disorders $(12 \%)$, genodermatosis $(10 \%)$, hypopigmentation disorders $(9.4 \%)$, neoplastic disorders (9\%), and hair disorders (7\%). Two-thirds of the publications were observational studies, and mostly case reports (44\%).

Conclusion: Dermatology research in SA has increased over the past decade. However, the quality of research remains inadequate. Saudi Arabia's dermatology research output is affected by the availability of funding and national research projects, which could improve the studies' quality. We recommend the establishment of data registry units that can aid researchers in producing high-quality studies, while encouraging the collaboration of different centers in various SA regions (and abroad) to conduct research with generalizable results.

Keywords: dermatology, publications, research, Saudi Arabia

Saudi Med J 2020; Vol. 41 (5): 524-531 doi: $10.15537 /$ smj.2020.5.25051

From the Dermatology Department, College of Medicine, King Saud University, Riyadh, Kingdom of Saudi Arabia.

Received 7th November 2019. Accepted 24th March 2020.

Address correspondence and reprint request to: Dr. Sarah Alsukait, Dermatology Department, College of Medicine, King Saud University, Riyadh, Kingdom of Saudi Arabia. E-mail: salsukait@gmail.com ORCID ID: https://orcid.org/0000-0002-6581-1475

$\mathrm{K}$ ingdom of Saudi Arabia (KSA) is the largest country in the Middle East, and among the largest top twenty countries in the world, extending more than 2 million kilometers in land area, with geographic, demographic, and economic advantages. ${ }^{1-3}$ The country continues to acknowledge the importance 
of research and the development of research centers, which is well-recognized through the 2030 vision lead by the Chairman of the Council of Economic and Development Affairs, Prince Mohammad bin Salman Al-Saud. ${ }^{4}$

Moreover, KSA has one of the fastest-growing higher education systems, having instituted a number of world-class universities and advanced technical institutes across the country. ${ }^{1,5}$ There are over 65 universities and degree-awarding institutions, with an educational budget of over $\$ 55$ billion for the year 2014. ${ }^{1}$ During the period from 1996 to 2012, SA has contributed more than 100,000 papers in the sciences and in medicine alone, with a total of 16,196 publications. ${ }^{6}$

It is well established that current medical practice is guided by evidence-based medicine (EBM) and an important element of medical training in SA is healthrelated research. Over $95 \%$ of resident physicians, who work in different specialties and regions of Riyadh, recognize the importance of research in health care; most are involved in a research at one point during their medical training. ${ }^{7}$

Although medical research in KSA is growing, compared to previous years, it cannot stand against other leading countries, such as the United States (USA) - ranked as the number one country in research activity. ${ }^{8,9}$ According to Scimago Journal \& Country Rank (SJR), which analyzed the number of publications per country and citations per document between 1996 and 2018, KSA was ranked 43rd and 42nd out of 239 countries in terms of documents published and citations, respectively; with a total of 182,753 documents published and 194,6422 citations. ${ }^{9}$

Bibliometric indicators are needed to provide an assessment of previously published data in SA, specifically dermatology-related publications, our field of study. This paper's aims are 2-fold; to i) analyze the quantity and characteristics of dermatologyrelated publications from the KSA and ii) determine the distribution of dermatology publications in KSA according to field or topic covered, type of study design, journal in which they were published (name and its impact factor), ethical status, year of publication, geographical location of the publication, authorshiprelated information (name and number of authors), and

Disclosure. Authors have no conflict of interests, and the work was not supported or funded by any drug company. affiliated institution. This study provides KSA's detailed analysis of dermatology related publications patterns.

Methods. This retrospective, descriptive study was conducted between October 2018 and July 2019 in the Dermatology Department, College of Medicine, King Saud University, Riyadh, KSA. We conducted a literature search to retrieve all dermatology-related publications in SA from the date of first published article in 1982 through December 31, 2018. Our extensive online search was performed utilizing the PubMed $^{\oplus}$ search engine (National Library of Medicine, Bethesda, Maryland, USA). PubMed was chosen because of its specialization in medical literature, and the use of its Boolean operator search capacity and its Medical Subject Headings (MeSH) terms; this improves probability of compiling the biggest number of relevant publications, with the large number of records available from publisher-supplied citations and the MEDLINE $^{\oplus}$ (National Library of Medicine) database. The search strategy included terms that are pertinent to the field of dermatology which were combined using the Boolean operators "and" or "or". We utilized the following MeSH terms: "Dermatology" AND "Saudi", "Cutaneous" AND "Saudi", and "Skin" AND "Saudi", with SA designated as the affiliated country.

We included all dermatology-related publications conducted in, and published from KSA, including international collaborative studies, with a primary author affiliated with a Saudi institution, and the study sample consisting of Saudi nationals or residents. Original articles, case reports or series, and reviews were included in our study. Whereas, book reviews, meetings' abstracts, communications, news items, letters, supplementary articles and editorials were excluded. In addition, articles published in languages other than English were excluded. Each included publication was analyzed comprehensively for the following data: title of the study, journal name, journal impact factor, citations' number, year of publication, geographical location in which the study was originated, location level, first author-related information (affiliated institution, name, gender, and specialty), collaboration pattern, study design, field of interest, ethical approval status, consent status, and funding support.

This study was approved by the Institutional Review Board (IRB) of the College of Medicine, King Saud University, Riyadh, KSA. Data were collected, stored, managed and coded in a spreadsheet using Excel $2010^{\circ}$ software. Data were analyzed using SPSS $^{\oplus}$ version 21.0 (IBM Inc., Chicago, Illinois, USA). Descriptive analysis was primarily carried out, where categorical 
variables were presented in the form of frequencies and percentages and continuous variables in the form of means. Several characteristics were further analyzed to show their distributions within some chosen subtypes.

Results. Based on our inclusion criteria, a total of 500 publications were selected for analysis. The first dermatological study conducted in SA was published in 1982. During the period from 1982 to 2018 , a gradual increase in the number of publications was observed in the first decade followed by a decrease in the subsequent decade (Figure 1). A clear rise in the rate of publications was seen between 2013 (17 papers/year) and 2018 (67 papers/year), with a slight drop in 2015. The average increase in this period was 13 articles per year. In our review, two-thirds of the studies were published within the last 9 years (2010-2018), while only 3\% of them were published in the first decade.

The annual cumulative number of citations based on Google Scholar, at the time of our data analysis (July 2019), is presented in Table 1. A fluctuation in the number of citations between the researched papers was observed. The highest number of citations per article was 304 for a study published in 2012, followed by 111 citations for articles published in 2002 and 91 in 1987.

Most studies were exclusively conducted in KSA, while only $7 \%$ were carried out in collaboration with other centers abroad. The proportion of regional publications versus the total number of national publications varied, with $52 \%$ from the central region, $24 \%$ from the eastern region, $12 \%$ from the western region, $6 \%$ from the southern region, 3\% from the northern region. We observed a decrease in collaborative work between the country regions, which account for only 3\% of the analyzed publications (Figure 2). Of the total number of publications, $24 \%$ stated that an IRB approved them and a similar percentage stated that informed consents were obtained from the participants. Only $13 \%$ of the published manuscripts were products of funded projects. Al-Qassim University, King Saud University and King Faisal Specialist Hospital and Research Center were the top 3 funding institutions, respectively.

The retrieved documents were published in different dermatological and non-dermatological indexed journals. Table 2 illustrates the most highly represented journals. The remaining journals had less than 10 publications each. The impact factors of these journals as listed in the Web of Science (2018) are shown in Table 3.

When the data were analyzed by field (Figure 3), the most researched field was infections, comprising $61(12 \%)$ articles. Of these studies, leishmaniasis was the most common subtype, with 24 studies published in the 1980s and 1990s. The second most-investigated field was genodermatosis, with $51(10 \%)$ studies. The first paper about a dermatological genetic disease was published in 1992, with the majority about cornification skin disorders and blistering diseases, including 15 papers each.

Although disorders of hypopigmentation represent the third most common field, with 47 publications $(9.4 \%)$, all of which were focused on vitiligo. The field of hyperpigmentation disorders comprised only 6 $(1.2 \%)$ studies.

Neoplastic diseases were the fourth most common field, with $43(9 \%)$ of the research studies. Published papers involve a variety of dermatological neoplasms

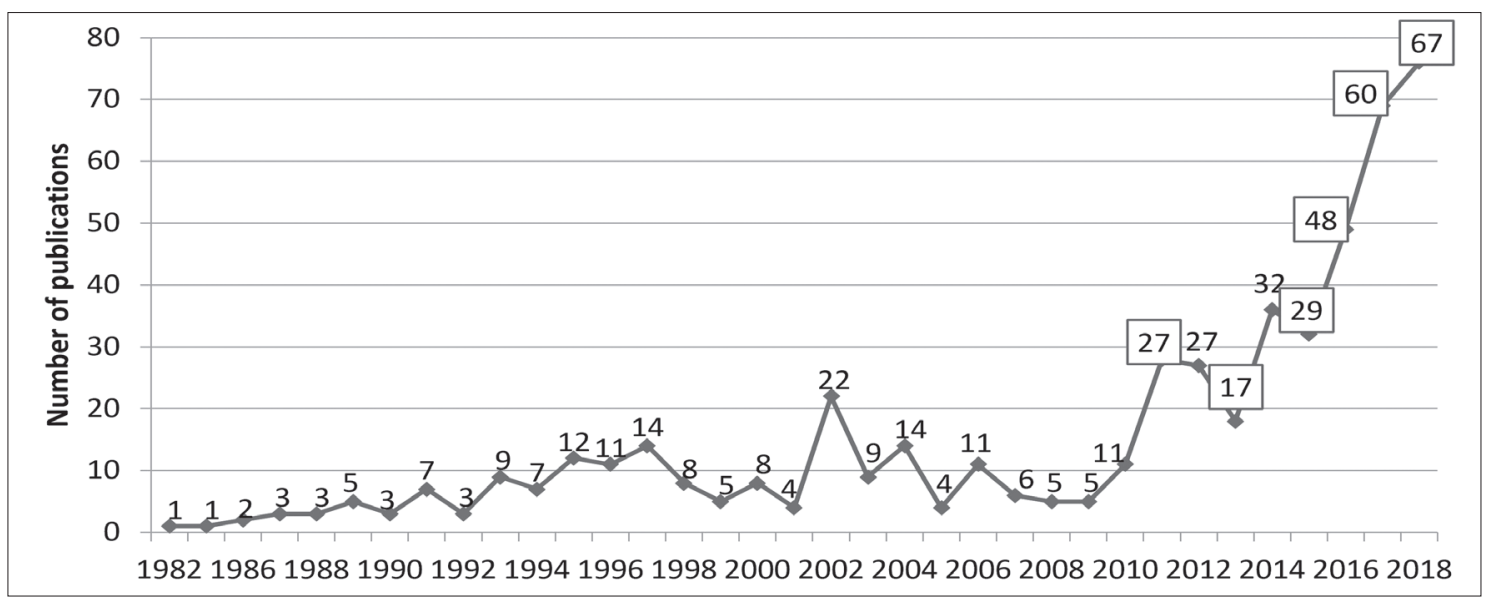

Figure 1 - Saudi dermatological publications pattern by year of publication $(\mathrm{N}=500)$. 
Dermatology publications in Saudi Arabia ... Alsaif et al

Table 1 - The annual number of publications and citations $(\mathrm{N}=500)$.

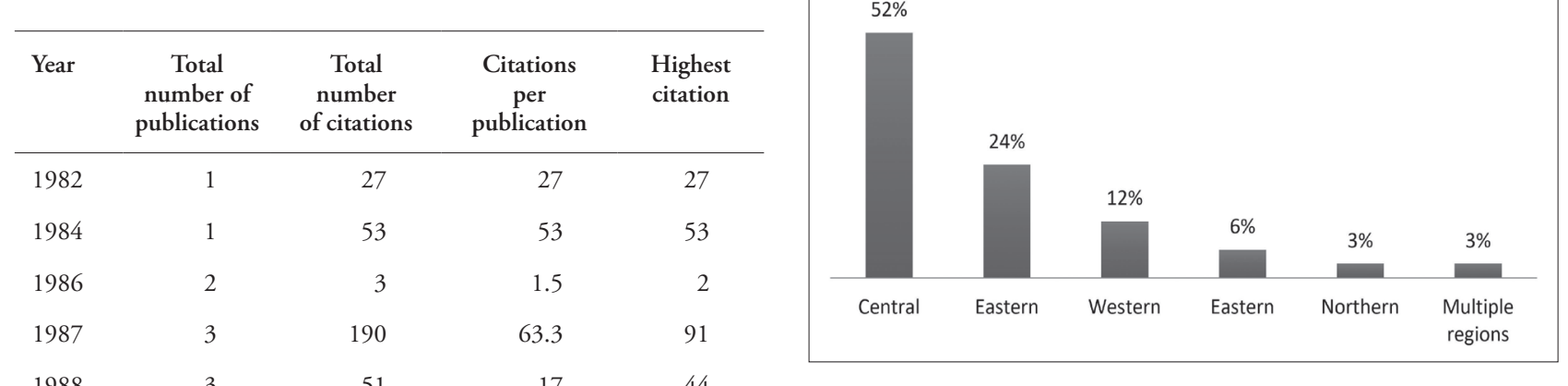

Figure 2 - Saudi dermatological publications pattern by study region $(\mathrm{N}=500)$.

$1989 \quad 5 \quad 160$

1996

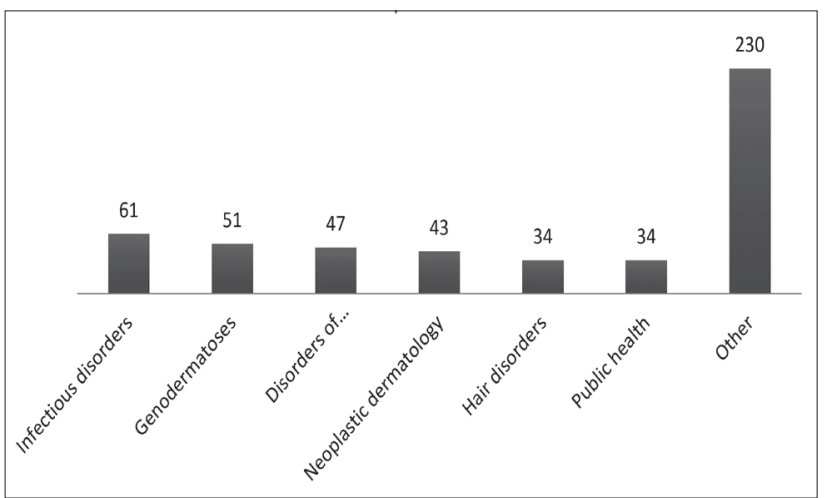

Figure 3 - Saudi dermatological publications pattern by study field $(\mathrm{N}=500)$.

Table 2 - Top 10 journals published dermatological studies from Saudi Arabia.

\begin{tabular}{lccc}
\hline Journal name & IF & n & $(\%)$ \\
\hline International Journal of Dermatology & 1.794 & 61 & $(11.9)$ \\
Annals of Saudi Medicine & 0.808 & 41 & $(7.8)$ \\
Saudi Medical Journal & 1.055 & 36 & $(6.8)$ \\
$\begin{array}{l}\text { Journal of the American Academy of } \\
\text { Dermatology }\end{array}$ & 7.102 & 26 & $(4.9)$ \\
$\begin{array}{l}\text { Journal of Cutaneous Medicine and } \\
\text { Surgery }\end{array}$ & 1.422 & 19 & $(4.0)$ \\
$\begin{array}{l}\text { Indian Journal of Dermatology, } \\
\text { Venereology, and Leprology }\end{array}$ & 3.030 & 19 & $(3.8)$ \\
Indian Dermatology Online Journal & NA & 18 & $(3.4)$ \\
$\begin{array}{l}\text { Pediatric Dermatology } \\
\text { International Journal of Health Sciences }\end{array}$ & NA & 12 & $(2.3)$ \\
Dermatologic Surgery Journal & 2.190 & 11 & $(2.1)$ \\
\hline$\quad$ IF: impact factor according to the Web of Science platform, 2018 \\
\hline
\end{tabular}




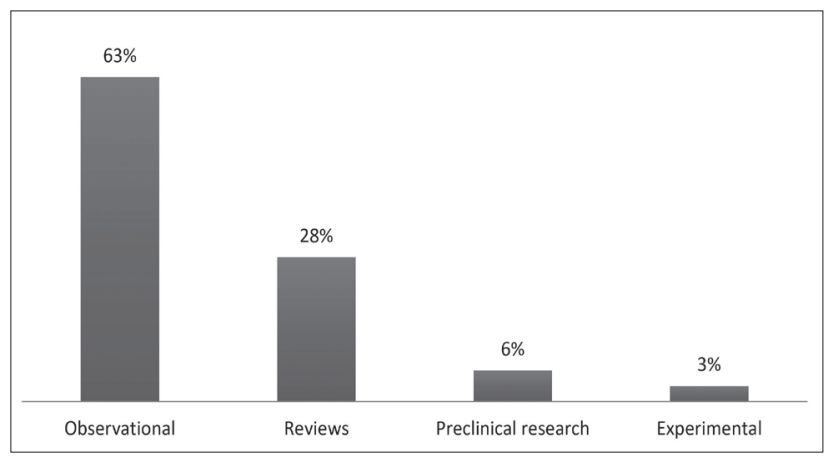

Figure 4 - Saudi dermatological publications pattern by study design.

such as vascular tumors, cutaneous T-cell lymphomas, adnexal, keratinocyte and benign melanocyte neoplasms. No study on melanoma was identified in our study.

Hair disorders were presented in 34 (7\%) publications, considered the fifth most common field. Half were relevant to alopecia areata. An equal number of publications was focused on dermatological public health, mainly studying the pattern of various skin diseases as well as patient awareness in different dermatological aspects.

Furthermore, other less frequently reported fields were analyzed in our study. Researches on papulosquamous disorders involved 24 articles; of these, 20 were about psoriasis. Therapeutics of different dermatological subjects were discussed in 24 published studies. Twenty-two were in the field of follicular occlusion disorders, and were divided into 19 articles on acne vulgaris, 2 articles on rosacea, and 1 article on hidradenitis suppurativa. A total of 16 articles were about eczematous disorders. Dermato-surgery field accounted for 13 published papers. Eight studies were published in lichenoid dermatosis, connective tissue disorders, and nail disorders. There were another 6 publications about autoimmune blistering disorders and 4 publications about different drug reactions, except Steven Johnson syndrome, which was not presented in our data. Other analyzed fields included depositional disorders (6 articles), dermatologic manifestations in patients with systemic diseases ( 4 articles), nutritional diseases (4 articles), psycho-cutaneous (4 articles), urticaria (3 articles), neutrophilic dermatosis (2 articles), panniculitis (2 articles), atrophic skin disorders (2 articles), and hypertrophic skin lesions (1 articles).

There were a variety of study designs, including 315 (63\%) observational studies, $140(28 \%)$ review papers, $30(6 \%)$ preclinical research and $15(3 \%)$ experimental studies (Figure 4). Of the observational studies, the majority were case reports (44\%) and cross-sectional studies (32\%), followed by case control (9\%), case series $(7 \%)$ and retrospective analysis $(6 \%)$, while only $2 \%$ were cohort studies. The reviews included literature reviews, a guideline about psoriasis and a single metaanalysis. The majority of preclinical studies were about drugs properties. Others included animal experiments, cell studies, and biochemical, genetic and physiological investigations. Of the experimental studies, 2 were randomized clinical trials.

Discussion. Assessing a particular field's research performance is important as it allows for recognition of a field's productivity and deficiencies in addition to establishing funding strategies and research programs, thereby improving research quantity and quality. In this study, we found it imperative to assess and analyze SA's dermatology research publications over the years to allow for such initiatives. Along with other tools, we have used mainly bibliometric indicators, since even with their weaknesses, they have become superior in assessing scientific research when used properly. ${ }^{10}$ Our study showed an overall deficiency in dermatology research. Nevertheless, the past several years has witnessed a substantial increase in the number of publications per year, which is in agreement with other articles assessing SA's overall research productivity. ${ }^{11,12}$ This could be attributed to several factors; instituting research hours as requirements for training programs acceptance, board certification, professional promotion by the Saudi Commission of Health Specialties and establishing the excellence allowance in universities. As $\mathrm{Meo}^{13}$ reported, SA has been going through a technological revolutionary period, developing highranking universities and research institutes, thereby transforming the country into a knowledge-based economy.

In terms of research quality, one of the methods used was to assess article influence on the literature is via citations number and impact factor of the publishing journal as. Although this is debatable, ${ }^{14}$ both are reasonable for quality assessment tools. ${ }^{15}$ In regard to citations, given our review, they averaged 18.19 per document. This is in contrast to the number of 8.67 citations/document as reported by the SCImago Journal and Country Rank (SCR), ${ }^{16}$ a portal that incorporates journal and the country's scientific indicators from the Scopus database. This could be explained by the fact that SCR's report includes articles from the year of 1996 only, and it is well-known that older articles tend to be more highly cited than recent ones, as they have 
had more time to acquire citations. ${ }^{17} \mathrm{On}$ a rare occasion though, the most cited article published by Alghamdi et $\mathrm{a}^{18}$ had 304 citations, close to being a citation classic; defined as an article with more than 400 citations. ${ }^{19}$

Moreover, assessing articles by the journal in which they are published, we found that journals with the highest published articles, in descending order of frequency, were the International Journal of Dermatology (11.9\% of articles) with an impact factor of 1.8, Annals of Saudi Medicine (7.8\% of articles) with an impact factor of 0.8 , and Saudi Medical Journal (6.8\% of articles) with an impact factor of 1.05 , indeed most of the publications were in low impact factor journals. Less less than $5 \%$ of the published articles were in the Journal of the American Academy of Dermatology, which has an impact factor of 7 and only 2 articles were published in JAMA Dermatology, the journal with the highest impact factor in the field of dermatology. Thus, it is evident that dermatology research in SA needs greater effort to improve its quality and its relevance. This is pointed out by Jamjoom ${ }^{20}$ who studied the relative international contribution of the scientific output by different specialties in KSA and deduced by his calculations that dermatology's output had a negative relative contribution. Furthermore, although publications were highest in an international journal (the International Journal of Dermatology), similar to Aboud et $\mathrm{al}^{21}$ who has reported that most of the publications from the Gulf Cooperation Council countries were in the International Journal of Dermatology. Yet it is worth mentioning that as both our searches included articles indexed in one search engine only. Therefore, we likely missed articles published by local journals in dermatology, which were not indexed in PubMed, including the Journal of the Saudi Society of Dermatology and Dermatological Surgery and this could have affected that statement.

Additionally, to assess quality, we examined whether articles attained Institutional Review Board (IRB) and consent from participants, if applicable. We have included these parameters as they indirectly infer quality. Investigators are required by IRBs to correct design flaws identified and to corroborate that studies are both valid and of value before receiving approval. ${ }^{22}$ Unfortunately, as mentioned in the Results section, only $24 \%$ of the publications stated that they were approved by an IRB, with a similar percentage documented that informed consent was obtained. This is alarming, as one of the ethical justifications to allow research with human subjects is gaining valuable scientific understanding, thereby promoting human health care. However, if there was no review committee to assess the validity of a study, the integrity of the results obtained may be unclear.

Another analysis of this study was the relative contribution of different regions in KSA to the number of dermatology publications. We found that the central region had the highest contribution of publications with approximately $52 \%$ of all articles. This may be due to the fact that the largest city (and capital of the country), Riyadh, is located in this region and has a high number of prestigious universities and hospitals. This finding corresponds with those of other articles on biomedical research in KSA by Latif, ${ }^{12}$ who reported $54.3 \%$ and Tadmouri et $\mathrm{al}^{23}$ who reported $69.9 \%$ of the total biomedical publications were from Riyadh. However, in terms of collaborative work in between regions, we found a significant deficiency, with only $3 \%$ of the articles were conducted as multiregional studies. Furthermore, only $7 \%$ of the studies were carried out in collaboration with other countries. These findings indicate that there is a great need for collaboration between regions, and with other countries to create more valuable research with results that can have a broader application to many populations.

An important finding of this review was the research gap in multiple dermatology disciplines. There was a paucity of epidemiologic studies. The prevalence of atopic dermatitis, psoriasis, lichen planus, vitiligo and other common disorders has yet to be determined in KSA. These types of studies are important to drive policy change, form guidelines, and initiate funding support-thereby allowing for more significant studies to be conducted. Substantial deficits remain in the understanding of many dermatological diseases and their treatments, necessitating further research in many areas. An example is psoriasis, which, even as one of the most commonly studied dermatoses worldwide, still has gaps in its research and care. ${ }^{24}$ In our review the first article concerned with psoriasis was long overdue being in being published in 2002. One of the unusual findings of our review was the rarity of studies on melasma and rosacea, both common complaints in the clinic. In the field of infections, Leishmaniasis in particular, had gained a great interest especially during the 1980 and 1990s. The majority of studies were conducted under the national Leishmaniasis research project, funded by King Abdulaziz City for Science and Technology, which may explain the high numbers. However, the project is now closed even though according to Abuzaid et $\mathrm{al}^{25}$ cutaneous Leishmaniasis is still endemic in KSA and that efforts should be made to fight it. This underscores the importance of research funds and their impact on the rapid acceleration of knowledge. 
This review showed that the bulk of studies were observational. Of these, case reports and case series prevailed, and although they advance our understanding and open up the field for new hypotheses, they are considered to have low-grade evidence. ${ }^{26}$ There was a lack of experimental studies. There are many opportunities to conduct trials at many fields of dermatology as it is a rapidly growing field with many new therapeutics. There was a similar lack of high quality cross-sectional studies, though they were high in number, as most had a convenient sample not a general-population based sample, thus, making the generalizability of the results poor.

Study limitations. This study is not without its limitations. It included electronically available online articles indexed in PubMed database only. We used particular $\mathrm{MeSH}$ terms that helped retrieve most published articles, relevant to our study; however, this might have missed some of them. Despite these limitations, we consider this study to be the first step towards in-depth analysis of dermatology research in KSA.

Many factors affect both quality and quantity of SA's dermatology research output. The shortage of Saudi dermatologists does not coincide well with the nation's health care goals, as suggested by Bin Saif et al. ${ }^{27}$ Other factors include deficits in funding support and lack of multiregional research collaborations, which would otherwise improve quality. As such, we recommend the development of clinical data registry units that can aid researchers in producing high-quality studies, and the collaboration of different centers in different regions in KSA (and abroad) to create research with generalizable results.

Acknowledgment. The authors would like to thank Deanship of Scientific Research (DSR) in King Saud University, Riyadh, Saudi Arabi for supporting this research through the initiative of DSR scholarship support. Also, we would like to thank the American Manuscript Editors (www.americanmanuscripteditors.com) for English language editing.

\section{References}

1. Meo SA. Saudi Arabia: A future regional hub for advanced education, research, science and technology. J Pak Med Assoc 2015; 65: 1112-1115.

2. National Industrial Clusters Development Program. (2019). Natural Resources [cited 2019; Accessed 2019 October 23]. Available from: https://www.ic.gov.sa/en/invest-in-saudiarabia/natural-resources

3. GIS Technology Innovation Center. The Role of Research Center for the Development of Saudi Arabia. [cited 2019; Accessed 2019 October 23]. Available from: http://www.gistic. org/the-role-of-research-center-for-the-development-of-saudiarabia/
4. Vision 2030. Saudi Vision 2030. [cited 2019; Accessed 2019 October 23]. Available from: https://vision2030.gov.sa/en

5. Top Universities. Study in Saudi Arabia. [cited 2018; Accessed 2019 October 23]. Available at: https://www.topuniversities. com/where-to-study/asia/saudi-arabia/guide

6. Meo SA, Hassan A, Usmani AM. Research progress and prospects of Saudi Arabia in global medical sciences. Eur Rev Med Pharmacol Sci 2013; 17: 3265-3271.

7. Mitwalli HA, Al Ghamdi KM, Moussa NA. Perceptions, attitudes, and practices towards research among resident physicians in training in Saudi Arabia. East Mediterr Health J 2014; 20: 99-104.

8. Alakloby OM. Dermatology research in Saudi Arabia: challenges and hopes. Journal of the Saudi Society of Dermatology \& Dermatologic Surgery 2012; 16: 53-55.

9. Scimago Journal \& Country Rank. SJR - International Science Ranking. [cited 2020; Accessed 2019 October 23]. Available at: https://www.scimagojr.com/countryrank. php?order=ci\&ord $=$ desc

10. Belter CW. Bibliometric indicators: opportunities and limits. $J$ Med Libr Assoc 2015; 103: 219-121.

11. Zaher WA, Meo SA, Almadi MA, Neel KF. Research productivity of health-care institutions of Saudi Government: Ten-year based bibliometric analysis. J Nat Sci Med 2018; 1: 13.

12. Latif R. Medical and biomedical research productivity from the Kingdom of Saudi Arabia (2008-2012). J Fam Community Med 2015; 22: 25-30.

13. Meo SA. Saudi Arabia: A future regional hub for advanced education, research, science and technology. J Pak Med Assoc 2015; 65: 1112-1115.

14. Gallagher EJ, Barnaby DP. Evidence of methodologic bias in the derivation of the Science Citation Index impact factor. Ann Emerg Med 1998; 31: 83-86.

15. Saha $S$, Saint $S$, Christakis DA. Impact factor: a valid measure of journal quality? J Med Libr Assoc 2003; 91: 42-46.

16. Scimago Journal \& Country Rank. SJR - International Science Ranking. [cited 2019; Accessed 2019 October 18]. Available from: https://www.scimagojr.com/countryrank.php?category= 2708\&order $=$ it\&ord $=$ desc

17. Wang J. Citation time window choice for research impact evaluation. Scientometrics 2013; 94: 851-872.

18. AlGhamdi KM, Kumar A, Moussa NA. Low-level laser therapy: a useful technique for enhancing the proliferation of various cultured cells. Lasers Med Sci 2012; 27: 237-249.

19. Martínez MA, Herrera M, López-Gijón J, Herrera-Viedma E. H-Classics: characterizing the concept of citation classics through H-index. Scientometrics 2014; 98: 1971-1983.

20. Jamjoom A. Medical speciality research in Saudi Arabia: A bibliometric assessment of productivity and worldwide ranking. J Health Spec 2017; 5: 23.

21. Al-Aboud FM, Al-Aboud KM, Al-Hawsawi KA, Ramesh V. Dermatological publications in the Gulf Cooperation Council countries. An analysis of 1966-2004 Medline papers. Saudi Med J 2004; 25: 1652-1655. 
22. Institutional Review Board Guidebook. Chapter IV Consideration of Research Design [cited 2019 October 19]. Available from: https://biotech.law.lsu.edu/research/fed/ohrp/ gb/irb_chapter4.htm

23. Tadmouri GO, Tadmouri NB. Biomedical research in the Kingdom of Saudi Arabia (1982-2000). Saudi Med J 2002; 23 : 20-24.

24. Ryan C, Korman NJ, Gelfand JM, Lim HW, Elmets CA, Feldman SR, et al. Research gaps in psoriasis: Opportunities for future studies. J Am Acad Dermatol 2014; 70: 146-167.
25. Abuzaid AA, Abdoon AM, Aldahan MA, Alzahrani AG, Alhakeem RF, Asiri AM, et al. Cutaneous leishmaniasis in Saudi Arabia: A comprehensive overview. Vector Borne Zoonotic Dis 2017; 17: 673-684.

26. McNair P, Lewis G. Levels of Evidence in Medicine. Int J Sports Phys Ther 2012; 7: 474-481.

27. Bin Saif GA, Al-haddab M. The Dermatology Workforce in Saudi Arabia: Current trends, challenges and future directions. Int J Health Sci 2010; 4: 178-193. 